\title{
Analysis of Wave Localization in a Disordered Periodic Viaduct Undergoing In-plane Vibration
}

\author{
Sheng Guo-jun Huang Qian \& XU Man-qing \\ Nanchang Institute of Technology, Nanchang, Jianxi, 330029, China
}

\begin{abstract}
KEYWORD: wave localization; periodic viaduct; Lyapunov exponent; disordered
ABSTRACT: Based on the wave transfer matrix method, wave localization in a disordered periodic viaduct undergoing in plane vibration is investigated according to the Wolf's algorithm in this paper. With the proposed model, the influences of the pier-height and beam-length disorders on the wave localization are examined. Also, the interactive effect of the damping and disorders on the wave localization in the disordered periodic viaduct is studied. Numerical results show that: in the pass-bands, for the assumed parameters of the viaduct, the localization due to the damping is larger than that due to the disorder. While in the stop-band, the viscosity of the materials tends to increase the Lyapunov exponent, while the disorder tends to diminish the Lyapunov exponent.
\end{abstract}

\section{INTRODUCTION}

The viaduct composed of the piers and adjacent horizontal beams is widely used as periodic structure to solve the settlement of soft ground. Because of the errors and material defects in the design and construction process, it is inevitably for periodic viaduct with a certain small range of deviation contrasting with the ideal periodic structure, which called disordered periodic viaduct. The research has shown that [1]: because of periodic structure disordering, the propagating waves or vibration will reflect at the node, which causes waves or vibration energy confined to a very small geometric range, forming local oscillation, this phenomenon is known as localized phenomenon. The wave localization damages to the rule of the periodic structure modal, in the action of external incentives, the localization will enlarge the response amplitude of some parts of the structure, resulting in the accumulation of energy, even leading to structural fatigue damage reducing the life of the structure. Thus, ignoring the disordered effects, still using the ideal periodic structure model to analyze the problem, it is possible to draw completely wrong conclusions. Therefore, it is important to research the wave localization in a disordered periodic viaduct for anti-seismic optimization design of the bridge structure.

The concept of localization was put forward earliest by Anderson [2] in the study of the disorder of solid state physics effects on metallic conductivity, which brought great interest. In the field of structural dynamics and vibration, the systematic research of elastic wave and vibration localization began in the early 1980s. For example, based on the similarity between the mechanical systems and solidstate physics system, Hodges [3] studied the vibration modes localization by the disordered structure. Kim [4] analyzed the substructure coupling stiffness and mass of multi-span periodic structure affected on modal localization. Using wave analysis method, Tan [5] analyzed the influence of modal localization of the chain structure and beam structure under the elastic constraints by the disordered beam-length and coupling stiffness. Kissel [6] pointed out the existence of vibration localization in multi-span disordered beam and calculated localized factor. It is studied the buckling mode localization in the fewer spans periodic plate girder structure using modal analysis in the documents [7,8]. Li Fengming etc. [9] studied the localized phenomenon of the dual-coupled disordered multi-span beams. Considering structural damping, Langley $[10,11]$ studied the joint effects of the damping and detuning on structural vibration modes localization in the one-dimensional periodic structure, Bouzit [12] studied the combined effect of the damping and detuning on structural dynamic characteristics in the spatial multi-span simply supported beam structure.

In summary, the localized phenomenon of periodic structure vibration in the field of structural dynamics was studied in more literature, but the research object is generally periodic structure composed of elastic beams. For periodic viaduct composed of beam-beam, beam- pier and the rigid junction between them, the localization analysis caused by disordered vibration in-plane is different from the periodic structure of elastic beam. At present, the localization analysis of disordered periodic viaduct has not been reported. In the thesis, based on the transfer matrix method, and using Wolf algo- 
rithm [13], the localization factor analysis of the viaduct axial and shear in-plane vibration undergoing the external excitation is investigated, combining with specific examples, the impact of the pier-height and beam-length and material damping on localized factors is discussed.

\section{CONTROL EQUATIONS OF PIER AND BEAM UNDERGOING IN-PLANE VIBRATION}

For the viaduct structure composed of pier, monolithic concrete track panels, rails and fasteners, simplifying the track panel, rail and fasteners of each span as horizontal beam in the physical model, so one period of viaduct concludes one pier and two horizontal beams, as shown in Fig.1.

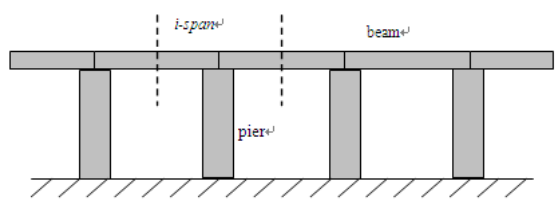

Fig.1 A schematic illustration of a periodic viaduct rigidly supported on a half space soil

Considering the axial and lateral vibration in the plane, for any span of pier and beam structure, according Bernouli-Euler beam theory [14], the vibration control equations of pier and beam in frequency domain can be expressed as,

$$
\begin{aligned}
& E_{s i} \frac{d^{2} u_{s i}(z)}{d z^{2}}-\rho_{s i} \omega^{2} u_{s i}(z)=0 \quad, \quad s=b, d \\
& E_{s i} I_{s i} \frac{d^{4} v_{s i}(z)}{d z^{4}}-\rho_{s i} A_{s i} \omega^{2} v_{s i}(z)=0
\end{aligned}
$$

where, it denotes the beam's equations when subscript $s$ is equal to $b$, it denotes the pier's equations when subscript $\mathrm{s}$ is equal to $\mathrm{d}, E_{s i}, \rho_{s i}$ and $A_{s i}$ are respectively elastic modulus, density, crosssectional area of piers and beams, $I_{s i}$ is cross-sectional rotational inertia, $u_{s i}(z)$ and $v_{s i}(z)$ are respectively axial and tangential displacement of piers and beams.

When No. i period structure of pier and beams is in-plane vibration of the axial and tangential direction, it can be obtained by the constitutive relation of Bernouli-Euler beam,

$$
\begin{aligned}
& N_{s i}(z)=E_{s i} A_{s i} \frac{d u_{s i}(z)}{d z}, \\
& Q_{s i}(z)=E_{s i} I_{s i} \frac{d^{3} v_{s i}(z)}{d z^{3}}, \\
& M_{s i}(z)=-E_{s i} I_{s i} \frac{d^{2} v_{s i}(z)}{d z^{2}}, \quad s=b, d
\end{aligned}
$$

The state vector $\Psi_{s i}(z)$ of arbitrary position $\mathrm{Z}$ is composed of displacement vector $\mathbf{q}_{s i}(z)$ and internal force vector $\mathbf{f}_{s i}(z)$, its expression is,

$$
\begin{aligned}
& \Psi_{s i}(z)=\left[\mathbf{q}_{s i}^{T}(z), \mathbf{f}_{s i}^{T}(z)\right]^{T}, \\
& \quad \mathbf{q}_{s i}(z)=\left[u_{s i}(z), v_{s i}(z), \theta_{s i}(z)\right]^{T}, \\
& \mathbf{f}_{s i}(z)=\left[N_{s i}(z), Q_{s i}(z), M_{s i}(z)\right]^{T}, \quad s=b, d
\end{aligned}
$$

According to the vibration control equation (1) of Bernouli-Euler beam and constitutive relation equation (2), the transfer matrix of pier and horizontal beam in the plane of vibration can be deduced.

$$
\boldsymbol{\Psi}_{b n}\left(\frac{L_{b n}}{2}\right)=\mathbf{T}_{b n}\left(\frac{L_{b n}}{2}\right) \mathbf{S}_{J n} \mathbf{T}_{b n}\left(\frac{L_{b n}}{2}\right) \boldsymbol{\Psi}_{b n}\left(-\frac{L_{b n}}{2}\right)
$$

It can be seen, for disordered periodic viaduct, considering the rigid connection between pier and beam, the beam transfer matrix of $\mathrm{n}$-span is as follows,

$$
\mathbf{T}_{c n}=\mathbf{T}_{b n}\left(\frac{L_{b n}}{2}\right) \mathbf{S}_{J n} \mathbf{T}_{b n}\left(\frac{L_{b n}}{2}\right)
$$


If the structure element of each span is same for viaduct, it can be obtained by Bloch theorem $[16]$,

$$
\boldsymbol{\Psi}_{b n}\left(\frac{L_{b}}{2}\right)=e^{-\mathrm{i} \kappa L_{b}} \boldsymbol{\Psi}_{b n}\left(-\frac{L_{b}}{2}\right)
$$

where, $\kappa$ is wave number of wave propagation in periodic structure.

Substituting equation (6) into equation (4), the characteristic equation of periodic viaduct can be obtained,

$$
\left[\mathbf{T}_{b n}\left(\frac{L_{b n}}{2}\right) \mathbf{S}_{J n} \mathbf{T}_{b n}\left(\frac{L_{b n}}{2}\right)-e^{-\mathrm{i} \kappa L_{b}} \mathbf{I}_{6 \times 6}\right] \boldsymbol{\Psi}_{b n}\left(-\frac{L_{b}}{2}\right)=0
$$

\section{LYAPUNOV EXPONENT OF DISORDERED PERIODIC VIADUCT}

If $\mathbf{X}$ is the feature vector of the transfer matrix $\mathbf{T}_{c n}$, there is,

$$
\boldsymbol{\Psi}_{i}=\mathbf{X V}_{i}, \quad i=n-1, n
$$

where, $\mathbf{V}_{n-1}$ ane $\mathbf{V}_{n}$ are respectively wave vector of n-span beam.

Without considering the disordering of the periodic viaduct, using equation (7), the following equation can be obtained by substituting equation (8) into equation (4)

$$
\mathbf{V}_{n}=\mathbf{X}^{-1} \mathbf{T}_{c} \mathbf{X} \mathbf{V}_{n-1}=\left[\begin{array}{lll}
\lambda_{1} & & 0 \\
& O & \\
0 & & \lambda_{6}
\end{array}\right] \mathbf{V}_{n-1}
$$

where, $j=1,2, \mathrm{~L}, 6, \lambda_{j}$ is the transfer matrix eigenvalue of $\mathrm{n}$-span beam.

\section{NUMERICAL ANALYSIS}

Using the proposed method, the wave localization by the disordering of the pier-height and beamlength is analyzed in the periodic viaduct undergoing in-plane vibration.

Fig. 2 shows respectively the positive Lyapunov exponents with disordering pier-height $(\delta=0.0$, $0.05,0.1$ ) at different frequencies. According to Fig.2 (a) and (b), only in a certain frequency range, the disordering pier-height impact on the first and the second positive Lyapunov exponents, but in the remaining frequency range, the disordering pier-height has almost no effect. From Fig.2 (c), in the frequency range of $25 \mathrm{~Hz} 35 \mathrm{~Hz}$, with the increase of $\delta$, the third positive Lyapunov exponent is reduced in the band gap, but in the pass band, the third positive Lyapunov exponent increases because of the localization of periodic viaduct.

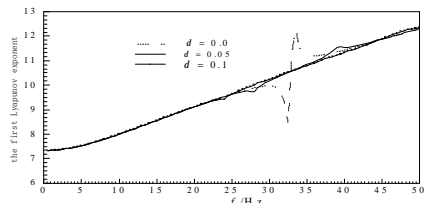

(a)

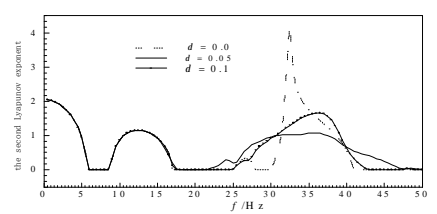

(b)

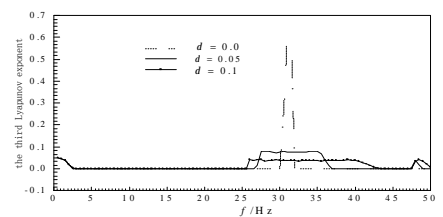

(c)

Fig. 2 The positive Lyapunov exponents versus frequency when disorder occurring in the pier heights 


\section{CONCLUSION}

Using the transfer matrix method, the wave localization of periodic viaduct in-plane vibration is analyzed, and the influences of the damping and the disordering of pier-height and beam-length on the wave localization are discussed. Numerical results show:

(1)The disordering of pier-height and beam-length has almost no effect on the Lyapunov exponent in the low frequency, with the increase of frequency, the phenomenon of the disordering periodic viaduct causing the localization becomes obviously, the main reason is smaller wavelength of the high frequency waves makes the structure sensitive with smaller disordering.

(2) Comparing with the first and second positive Lyapunov exponent, the third positive Lyapunov exponent is smaller, so the third positive Lyapunov exponent can reflect the wave decay of periodic structure, namely localization factor.

\section{ACKNOWLEDGMENTS}

The project is supported by the National Natural Science Foundation of China with grant number No. 51269021, 51569016. Also, the research is supported by the Jiangxi province science and technology plan projects(No: 20133ACB20006, 20141BBG70088). Moreover, the financial support from Science and technology project of Jiangxi Provincial Education Department Number GJJ14755 is greatly appreciated.

\section{REFERENCE}

[1] Li Fengming Hu Chao Huang Wenhu Zhou Min Wave localization in disordered multi- span beams with elastic supports[J]. ACTA MECHANICA SOLID SINICA, 2004, 25(1): 83-86

[2] Anderson PW Absence of diffusion in certain random lattices[J]. Physical Review, 1958, 109(5): 1492-1505.

[3] Hodges CH Confinement of vibration by structural irregularity[J]. Journal of Sound and Vibration, 1982, 82(3): 411-424.

[4] Kim DO, Lee IW Mode localizations in structures consisting of substructures and couplers[J]. Engineering Structures, 2000, 22: 39-48.

[5] Tan CA, Riedel CH Wave analysis of mode localization and delocalization in elastically constrained strings and beams[J]. Journal of Vibration and Acoustics, 1999, 121:169-173.

[6] Kissel GJ Localization in disordered periodic structures[D]. 1988.

[7] Elishakoff I, Li YW, Starnes Jr JH Passive control of buckling deformation via Anderson localization phenomena[J]. Chaos, Solitons \& Fractals, 1997, 8(1): 59-75.

[8] Li YW, Elishakoff I, Starnes Jr JH Buckling mode localization in a multi-span periodic structure with a disorder in a single span[J]. Chaos, Solitons \& Fractals, 1995, 5(6): 955-969.

[9] Li Fengming $\mathrm{Hu}$ Chao Huang Wenhu Elastic wave localization in one-dimensional periodic wave-guides[J].Chinese Journal Of Applied Mechanics , 2002, 19(4): 47-51).

[10] Langley RS Mode localization up to high frequencies in coupled one-dimensional subsystems[J]. Journal of Sound and Vibration, 1995, 185(1): 79-91.

[11] Langley RS On the forced response of one-dimensional periodic structures: vibration localization by damping[J]. Journal of Sound and Vibration. 1994, 178(3): 411-428.

[12] Bouzit D, Pierre C Localization of vibration in disordered multi-span beams with damping[J]. Journal of Sound and Vibration, 1995, 187(4): 625-648.

[13] Wolf A, Swift JB, Swinney HL, Vastano JA Determining Lyapunov exponents from a time series[J]. Phys. D Nonlinear Phenom. 1985, 16, 285-317.

[14] Graff KF Wave Motion in Elastic Solids[M]. Dover Publication, New York, 1991.

[15] Carcione J Wave Fields in Real Media-Wave Propagation in Anisotropic, Anelastic and Porous Media. Pergamon, Amsterdam (2001)

[16] Kittel C Introduction to Solid State Physics[M]. Wiley, New York, 1996. 